\title{
Detection, Characterization, and Screening of Heme-Binding Molecules by Mass Spectrometry for Malaria Drug Discovery
}

Katalina Muñoz-Durango, ${ }^{\dagger, \ddagger, \perp}$ Alexandre Maciuk, ${ }^{* \dagger}{ }^{\dagger}$ Abha Harfouche, ${ }^{\dagger}$ Sandra Torijano-Gutiérrez, ${ }^{\dagger, q}$ Jean-Christophe Jullian, ${ }^{\dagger}$ Jérôme Quintin, ${ }^{\dagger, \#}$ Kevin Spelman, ${ }^{\dagger, \|}$ Elisabeth Mouray, ${ }^{\S}$ Philippe Grellier, ${ }^{\S}$ and Bruno Figadère* ${ }^{*} \dagger$

${ }^{\dagger}$ Laboratoire de Pharmacognosie, UMR 8076 CNRS BioCIS, Faculté de Pharmacie, Université Paris-Sud, 5 rue J.-B. Clément, 92296 Châtenay-Malabry, France

${ }^{\ddagger}$ Grupo de Investigación en Sustancias Bioactivas, Sede de Investigaciones Universitarias, SIU. Car. 53 \# 61-30, Laboratory 229, Universidad de Antioquia, Medellin, Colombia

${ }^{\S}$ UMR 7245 CNRS MNHN, Molécules de Communication et Adaptation des Micro-organismes, Museum National d'Histoire Naturelle, 61 rue Buffon, 75005 Paris, France

Supporting Information

ABSTRACT: Drug screening for antimalarials uses heme biocrystallization inhibition methods as an alternative to parasite cultures, but they involve complex processes and cannot detect artemisinin-like molecules. The described method detects heme-binding compounds by mass spectrometry, using dissociation of the drug-heme adducts to evaluate putative antiplasmodial activity. Applied to a chemical library, it showed a good hit-to-lead ratio and is an efficient early stage screening for complex mixtures like natural extracts.

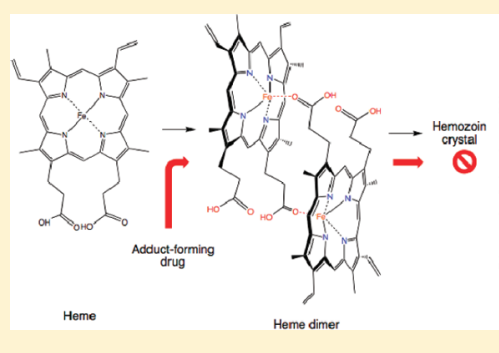

Artemisinin-heme adduct stability in collision-induced dissociation MS

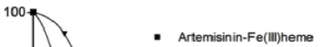
Artemisinin-Fe(II)heme

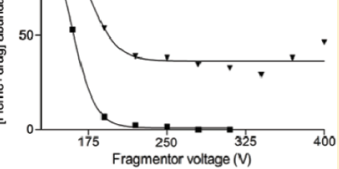

$\mathrm{M}$ alaria is a recurring challenge for health policies, medicinal research, and the pharmaceutical industry. This potentially lethal infection is vector-borne and thrives in tropical areas that are often highly populated by low-income communities resulting in the endemic exposure of 3 billion people to the various species and strains of Plasmodium. The fact that most of the drugs currently used to cure or prevent malaria have been known and used for decades (if not centuries) also contributes to the critical situation in terms of resistance. The emerging resistance to artemisinine analogs is of primary concern. Antimalarial drug discovery can follow two approaches. Assays performed on whole parasite in living erythrocytes have the advantage of allowing the discovery of drugs acting on unidentified targets but imply the use of blood of human or animal origin, along with handling of live, infective parasites. ${ }^{1}$ Target-based approaches require much simpler equipment. One of the most studied and most promising targets in malaria is the strictly parasite-specific, heme detoxification pathway. The massive release of free, toxic ferriprotoporphyrin IX is handled by the parasite via its biomineralization into hemozoin, a supramolecular assembly of heme dimers (Figure 1). ${ }^{2,3}$ As it is not protein dependent, there is no possibility of resistance against this target per se by genotypic mutation or phenotypic overexpression. Consequently, the inhibition of synthetic hemozoin formation (called $\beta$-hematin) is the basis for a variety of tests aimed at the discovery of antimalarial compounds. ${ }^{4}$ Noteworthy, the

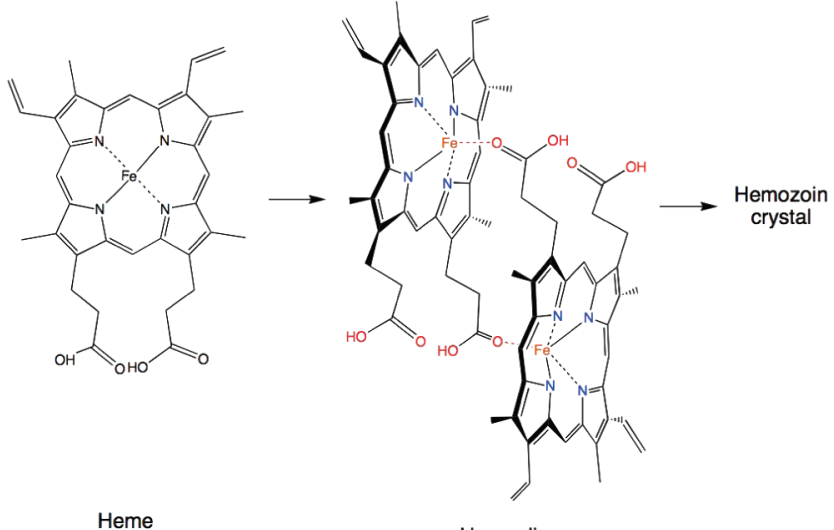

Heme dimer

Figure 1. Hemozoin formation pathway.

hemozoin pathway can also be considered as a target for antischistosomal compounds. ${ }^{5}$

The use of mass spectrometry to confirm and characterize complexes between small molecules and $\mathrm{Fe}$ (III) heme has been used by several authors. ${ }^{6-10}$ Some of these authors used collision-induced dissociation (CID) to assess the strength of the association between $\mathrm{Fe}$ (III) heme and drugs. In the present

Received: January 8, 2012

Accepted: March 2, 2012

Published: March 2, 2012 
work, we validate and implement this approach for a complex mixture. A chemical library has also been screened to demonstrate it can be used as a screening tool, especially for natural products and extracts. We also set up a protocol identifying compounds that form a covalent bond with $\mathrm{Fe}$ (II) heme, providing an original screening tool able to detect artemisinine-like compounds.

\section{EXPERIMENTAL METHODS}

Hemin, the ferriprotoporphyrin used for the incubation, gave several molecular ions in the simple quadrupole instrument, namely, $[\mathrm{Heme}]^{+}$at $\mathrm{m} / z 616.2$ and heme dimers at $\mathrm{m} / z 1232.3$ $\left([2 \mathrm{Heme}]^{+}\right)$and $m / z 1253.3\left([2 \mathrm{Heme}+\mathrm{Na}]^{+}\right)$. The characteristic isotopic pattern of heme (mainly, $\mathrm{M}+1$ for $39.6 \%$ along with $M+2$ for 9.2\%) makes the signal of any species containing heme clearly identifiable. Heme is detected at its native mass although the iron is in the $\mathrm{Fe}$ (III) form, as it is complexed by the porphyrin nitrogens. Consequently, Fe(II) heme is not detected by MS. Heme dimers were observed in the triple quadrupole spectra except the dimer-sodium adducts. The specificity of the method, i.e., its ability to detect hemetargeting entities, was validated on a set of compounds showing antimalarial activity, acting by heme disposal interference (positive controls) or by other mechanisms (negative controls). Adducts were obtained with a large set of antimalarial drugs tested (Table 1 in Supporting Information). In addition to the identification of adducts, a relative indication of the strength of the binding between two components of a complex can be obtained by mass spectrometry. Indeed, in a single quadrupole, the parameter called fragmentor voltage is the transfer voltage applied between the end of the transfer capillary and the first skimmer placed in front of the focusing octopole and the quadrupole analyzer. The higher the voltage, the faster ions are accelerated and the higher the energy of subsequent intermolecular collisions. In such an instrument, fragmentor voltage also impacts the ion transfer. As a consequence, for a given species subjected to fragmentor variations, abundance variations are the result of transfer variations as well as fragmentation if any. To account for these effects, we recorded the abundance of heme depending on the fragmentor voltage, using a flow injection analysis mode consisting of repeated sample injections at increasing fragmentor voltage, ranging from 130 to $400 \mathrm{~V}$ with $30 \mathrm{~V}$ increments. Abundance intensities were recorded and plotted as a function of fragmentor voltage, the maximum abundance being normalized at $100 \%$. The fragmentor voltage determining $50 \%$ of the dissociation is noted as $\mathrm{DV}_{50}$.

A fragmentor increase in simple quadrupole instrument causes a decrease of heme abundance signal, until a reproducible plateau of $41 \%$ that can be considered as the amount of heme molecule resistant to fragmentation and enventually transferred to the analyzer (Figure 2). Indeed, in such collision-induced dissociation (CID) experiments, intramolecular fragmentations are less likely to appear, as they require more energy and as the ESI mode used in these experiments are designed to prevent molecular fragmentation. In the triple quadrupole instrument, the acceleration is performed by the second quadrupole and collisions with the inert gas are much more energetic, finally leading to a complete disappearance of the heme signal without any plateau.
Heme stability in simple quadrupole MS

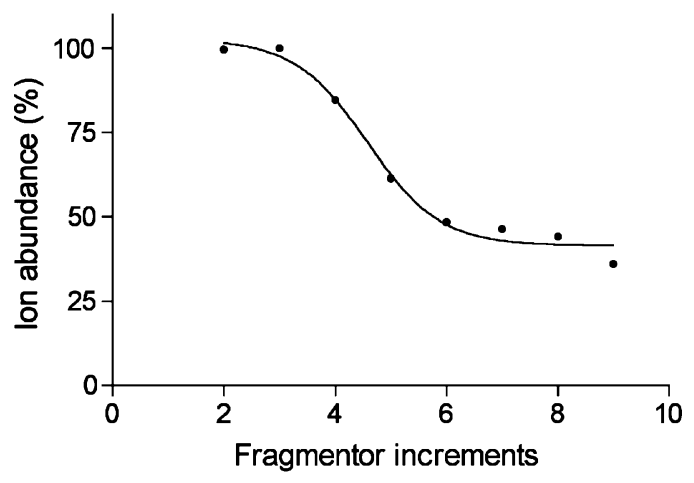

Heme stability in triple quadrupole MS

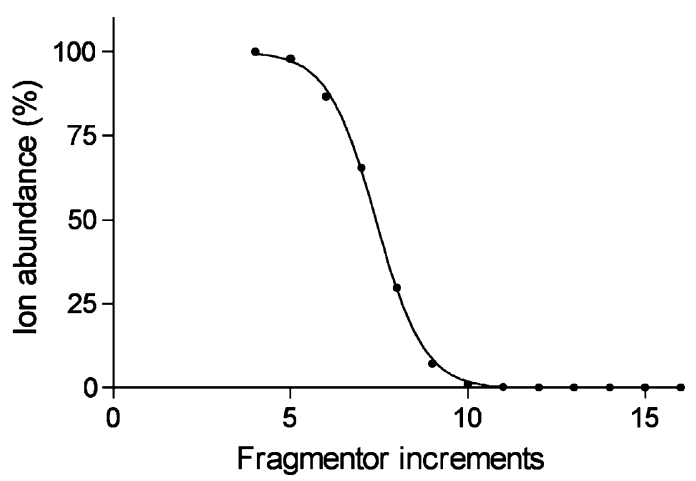

Figure 2. Stability curves of heme $(m / z=616)$ obtained by CID experiments in simple quadrupole and triple quadrupole instrument (fragmentor conditions: see text).

\section{RESULTS}

CID experiments were performed for heme-drug adducts, and examples of curves are given in Figure 3 (curve data are

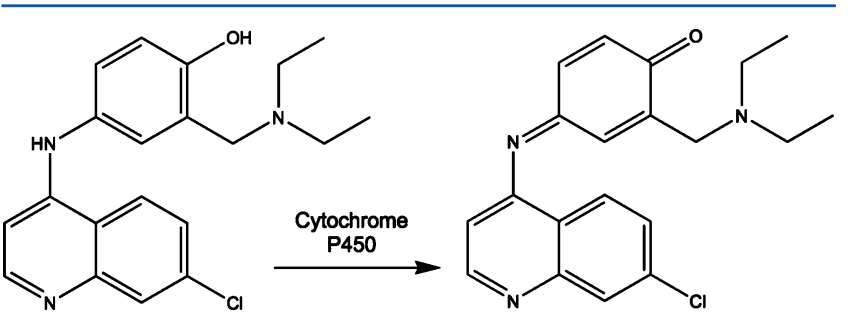

Figure 3. Amodiaquine metabolism providing a quinone-imine.

summarized in Table 1 of Supporting Information). Compounds like quinolines, artemisinine, ${ }^{11,2}$ azoles, ${ }^{12,13}$ halofantrine, and pyronaridine are well documented for acting via a heme-interacting mechanism.

Among quinolines, only amodiaquine and piperaquine show a significantly different curve than every other quinoline. Although the $\mathrm{DV}_{50}$ is similar, a significant amount of complex (ca 8\%) remains present even for higher fragmentor voltages. This can be interpreted as the consequence of a particularly strong binding between amodiaquine and heme. Among quinolines, amodiaquine shows enhanced activity, parasitespecific accumulation, and decreased cross-resistance pattern in vitro. Its structure comprises $p$-hydroxyaniline aromatic ring, substituted at $5^{\prime}$ with an alkylamine, and has been under focus 
of several structure-activity relationship (SAR) studies. Studies on the interaction of amodiaquine and heme have demonstrated that the aromatic ring increases lipophilicity and side chain rigidity, thus favoring $\pi-\pi$ stacking interactions (ref 14 and references cited therein). Moreover, the 5 ' position allows modulation of the lipophilicity, having a significant effect on activity and cross-resistance. The importance for the high activity of amodiaquine of the 4'-hydroxy group involved in a Mannich-base pattern is controversial but does not seem to be crucial for activity, as 4'-dehydroxy amodiaquine maintains and even shows higher antimalarial activity. Noteworthy, pyronaridine also contains a Mannich base pattern, but it is hindered by two adjacent pyrrolidine, explaining its lower reactivity. However, it has been shown to be a substrate of oxidation by cytochromes P450 leading to a quinone-imine, an electrophilic metabolite susceptible to bind to proteins and contributes to its toxicity (Figure 3). ${ }^{14}$

These data corroborate our findings, suggesting a particularly strong interaction between amodiaquine and heme that could be constituted by a covalent bound. Traditional $\beta$-hematin inhibition assays comparing chloroquine and amodiaquine do not indicate a higher $\beta$-hematin polymerization inhibition by amodiaquine, whereas it has a significantly higher activity in vitro and in vivo. Thus, besides its ability to evaluate the hemedrug complex binding strength, our method may be a more relevant tool to screen rapidly for putative activity of molecules. On the other hand, piperaquine does not present any group susceptible to form covalent bounds, but its lipophilicity is significantly higher than other quinolines $(\log P=6.79$ vs 3 to 5 range, respectively). Such hydrophobicity may explain a strong hydrophobic interaction between piperaquine and heme. As far as the $\mathrm{DV}_{50}$ value of curves is concerned, it can be considered as an indication of the relative affinity toward heme, putatively correlated to the ability of drugs to inhibit $\beta$-hematin formation. Indeed, within the quinoline group, the relative ranking of drug affinities for heme in our method is consistent with the $\mathrm{IC}_{50}$ ranking obtained with $\beta$-hematin inhibition methods as reported in the literature ${ }^{15}$ (when available from one homogeneous experiment set), namely, amodiaquine > quinidine $>$ cinchonidine $>$ mefloquine.

Antifungal azoles are known to exert their antimalarial activity through inhibition of hemozoin formation. ${ }^{13}$ Hybrid drugs combining 4-aminoquinoline- and clotrimazole-based pharmacophores have been synthesized and showed higher $\beta$ hematin polymerization inhibition than chloroquine or clotrimazole by themselves. ${ }^{16}$ As expected, every azole tested in the present method formed an adduct with heme.

Relative stabilities of heme-drug adducts showed significant differences between tested azoles. When considering $\mathrm{DV}_{50}$ as a relative indication of stability, complex stability can be ranked by increasing stability as miconazole < clotrimazole < ketoconazole. This is in accordance with data of $\mathrm{IC}_{50}$ for $\beta$ hematin polymerization inhibition reported by Chong et al., ${ }^{12}$ namely, ketoconazole $>$ clotrimazole $>$ miconazole. Binding of heme with azoles was suggested in our method to be stronger than with quinolines, which is not in accordance with the biological activities for the azole series. This may be explained by lipophilicity characteristics and $\mathrm{p} K_{\mathrm{a}}$ values that may reduce the ability of the drug to reach the digestive vacuole, as well as the accumulation phenomenon within it, which is partly responsible for the high activity of some quinolines.

Antibiotics like doxycycline and clindamycin are good candidates for combined antimalarial therapies because of their delayed parasiticidal action on the apicoplast. ${ }^{17}$ Biguanide derivatives (proguanil, metformine, and phenformine) are used as antimalarial or antihyperglycemic agents, but the mechanism of antimalarial action is still unclear and hypothesized to be a dihydrofolate reductase inhibition, along with a protease inhibition and the mitochondrial complex I inhibition. Cyclines and biguanides have not been described as drugs inhibiting $\beta$ hematin polymerization, but most of them have never been assessed for such activity. Nevertheless, one common feature of these molecules may be their binding to endogenous metals, e.g., $\mathrm{Zn}^{2+}, \mathrm{Cu}^{2+}$, and $\mathrm{Fe}^{3+18,19}$ that can explain the formation of adducts with heme in our protocol.

Other therapeutical classes are known to possess antimalarial activity without being described inhibitors of $\beta$-hematin formation: among them, antifolates (pyrimethamine, sulfadoxine) act by interfering with the folate metabolism essential to the parasite. ${ }^{20}$ Triclosan, a chlorinated antiseptic, interferes with the parasite-specific fatty acid synthesis in the apicoplast, along with other mechanisms of action. ${ }^{21}$ Dinitroaniline antimitotic herbicides (trifluralin, oryzalin) target the parasite-specific region of tubulin and showed antimalarial activity and no mammalian cell toxicity. ${ }^{22}$ When these compounds were incubated with heme, no adduct or adducts of very weak stability $\left(\mathrm{DV}_{50}<200 \mathrm{~V}\right.$, see below) were observed. For example an adduct was observed for sulfadoxine which contains a sulfur atom that may explain some affinity with heme. Indeed, sulfur and iron are known to form clusters, and most of the mitochondrial electron transport enzymes are based on ironsulfur clusters. ${ }^{23}$ Adduct with pyrimethamin may be based on the ability of the diaminopyrimidine group to interact with heme in a coordination mode.

Artemisinin and its derivatives are known to act by alkylating heme and proteins following a chemical change catalyzed by $\mathrm{Fe}$ (II) heme. As demonstrated by Robert et al., ${ }^{24}$ the reductive activation of endoperoxide by $\mathrm{Fe}(\mathrm{II})$ heme produces the homolytic cleavage of the endoperoxide bond and the subsequent formation of drug-derived C-centered radicals, able to alkylate heme or other proteins. The covalent binding of artemisinin to heme is a determining element explaining its significant activity.

In order to specifically identify compounds acting like artemisinin, an incubation was performed with $\mathrm{Fe}(\mathrm{II})$ heme generated in situ by adding glutathione $2.5 \mathrm{mM}$ in the incubation mixture as described by Robert et al. ${ }^{24}$ Such conditions reproduce the content of the erythrocyte where the high concentration of this mild reducing agent devoted to protect parasite from oxidative stress (redox milieu $-250 \mathrm{mV}$ ) contributes to prevent $\mathrm{Fe}$ (II) heme oxidation. In our method, artifacts specific to these reductive conditions could be observed in the spectra, mostly species at $\mathrm{m} / z 613$ [glutathione oxidized dimers $]^{+}$and $m / z$ 694.2 [heme + DMSO $]^{+}$. The absence of signal at $\mathrm{m} / z 616.2$ confirmed the complete reduction of heme into its $\mathrm{Fe}$ (II) form, as $\mathrm{Fe}$ (II) heme does not ionize in this ionization source. When artemisinin is incubated with $\mathrm{Fe}$ (III) heme, our method shows that the adduct formed by artemisinin is weaker than the heme-quinolines adducts $\left(\mathrm{DV}_{50} 160 \mathrm{~V}\right.$ and $170-204 \mathrm{~V}$ range, respectively). However, when incubated under reductive conditions and therefore with $\mathrm{Fe}$ (II) heme, artemisinin forms an adduct of higher affinity $\left(\mathrm{DV}_{50} 177 \mathrm{~V}\right)$ and is resistant to fragmentation even at high voltage, as can be seen on the plateau of $36.5 \%$, suggesting an adduct as stable as hemin itself (see Figure 4). Such a pattern is consistent with the covalent nature of the binding between 


\section{Relative stabilities of heme-drug adducts}

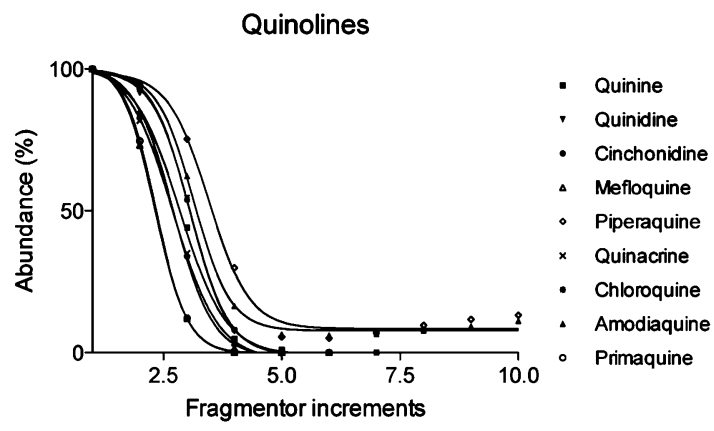

Azoles

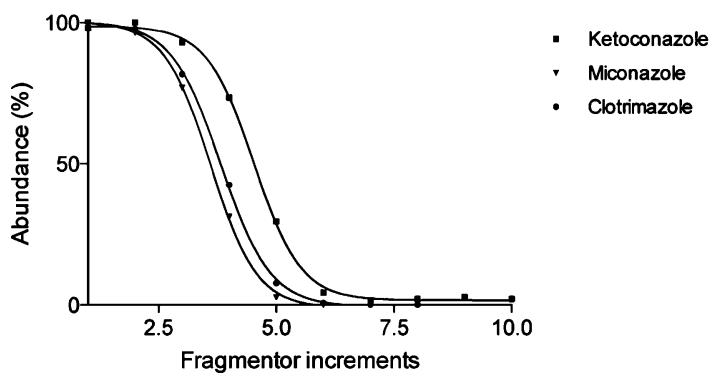

Artemisinin

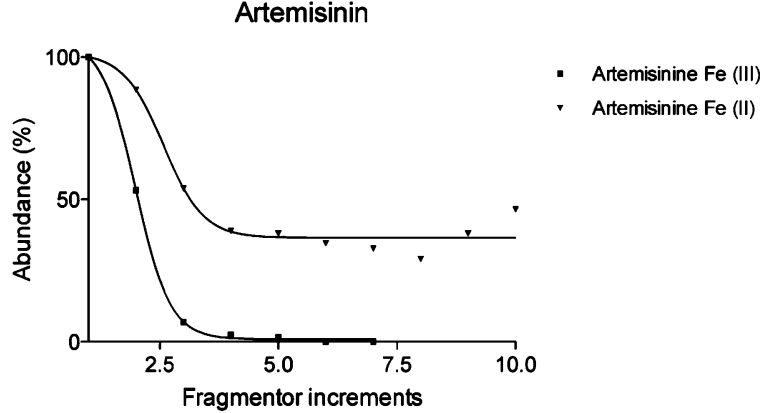

Figure 4. Stability curves obtained by CID experiments performed on the different heme-drug complexes. Fragmentor values range from $130(x=1)$ to $400 \mathrm{~V}(x=10)$ with $30 \mathrm{~V}$ increments. Adducts observed for pure standard drugs and $\mathrm{Fe}(\mathrm{III})$ heme if not otherwise stated.

heme and artemisinin. The covalent nature of the binding between the adducts of artemisinin with $\mathrm{Fe}(\mathrm{II})$ heme was confirmed by the appearance in the spectrum of the species at $m / z 898.5$ but also at $m / z 870.4, m / z 855.4$, and $m / z$ 838.4, resulting from fragmentations specific to the covalent adduct as reported by Accardo et al. ${ }^{25}$

Triple quadrupole CID experiments corroborated these observations. Indeed, the decreasing signal at $\mathrm{m} / \mathrm{z} 898$ corresponding to artemisinin-Fe(II) heme could be correlated with the increasing signal at $m / z 838$, corresponding to the loss of $\left[\mathrm{CH}_{2}-\mathrm{COOH}+\mathrm{H}\right]^{+}$as was reported by Robert et al., ${ }^{24}$ thus confirming the stable bound between artemisinin and heme (Figure 5)

The ability of the method to detect adduct-forming compounds in complex mixtures was assessed by incubating $\mathrm{Fe}(\mathrm{III})$ heme with natural extracts, namely, Cinchona bark alkaloidic extract and Artemisia extract. Cinchona alkaloidic extract contains a mixture of naturally occurring quinoline

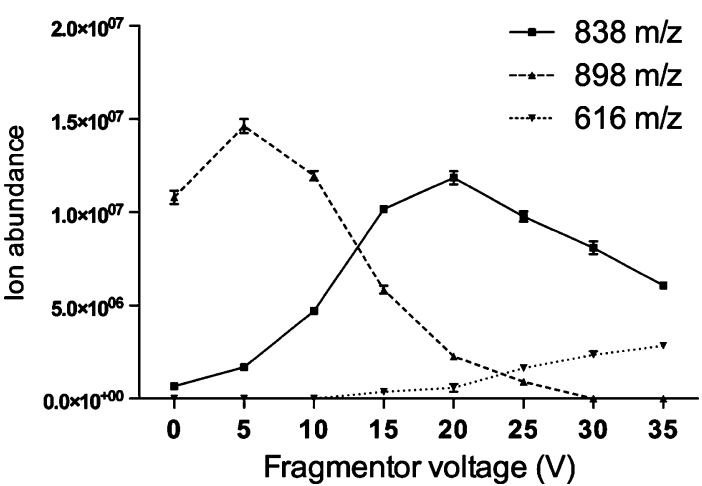

Figure 5. Effect of fragmentor voltage in triple quadrupole $\mathrm{MS}^{2}$ on abundances of artemisinine-heme adduct and fragments thereof (experimental conditions: see text).

alkaloids in various proportions, mainly, quinine, quinidine, cinchonine, and cinchonidine along with other secondary metabolites. Mass spectra of heme-Cinchona showed peaks at $\mathrm{m} / \mathrm{z} 295.0$ ([cinchonine/cinchonidine $+\mathrm{H}]^{+}$) and $\mathrm{m} / \mathrm{z} 325.0$ ([quinine/quinidine $]^{+}$) and adducts at $\mathrm{m} / z 910.3$ ([cinchonine/cinchonidine + heme $]^{+}$) and $\mathrm{m} / z$ 940.3 ([quinine/ quinidine + heme $]^{+}$) (See Figure 2A in Supporting Information). Artemisia cyclohexane extract shows greater complexity, but mass spectra of heme-Artemisia showed peaks of artemisinin-heme adducts fragments at $\mathrm{m} / z 870.4$ and 855.4 (See Figure 2B in Supporting Information). Noteworthy, the stability curve of artemisinin-Fe(II) heme adducts can be obtained from adduct detected in the extract and is similar to the curve obtained with artemisinin standard (data not shown). The method is therefore able to specifically detect antimalarial compounds based on a covalent interaction, which is a major factor of clinical efficacy.

\section{DISCUSSION}

Currently, a dozen of antimalarial molecules only are in the development stage out of the $>5$ millions compounds screened so far in various programs. Such an attrition rate highlights the need of high-throughput techniques for antimalarial drug discovery. Beyond this prerequisite, the path to such compounds can follow two approaches. ${ }^{4}$

Assays performed on whole parasite in living erythrocytes have the advantage of allowing the discovery of drugs acting on unidentified targets and are favored by companies. Several monitoring strategies are used to measure parasite replication in red blood cells. The most sensitive method is based on the incorporation of ${ }^{3} \mathrm{H}$-hypoxanthine by the parasite. Radioactivity handling is renowned to be expensive (probe purchasing and disposal) and uprises safety and environmental concerns. Parasites can alternatively be labeled by fluorescent probes and counted by flow cytometry. These dyes are mutagenic and cause low signal-to-noise ratios due to their binding to exogenous (human) DNA. Colorimetric monitoring is possible but requires many steps and expensive reagents. Transgenic parasites allow luminescence assays (through transfected luciferase), but these strains still carry drug selectable markers, which might interfere with the drug screening process. Some of these tests can be performed in microplates, but none seem to have been used in a high-throughput screening (HTS) campaign. In all cases, culture is performed in red blood cells, implying the use of blood of human or animal origin, along with alive, infective parasites handling. 
Target-based approaches require much simpler equipment. The hemozoin formation pathway is a major target, and efforts to reproduce heme crystallization outside the parasite have led to a vast literature. Dimers associate in a crystal called $\beta$ hematin, which is identical to hemozoin (Figure 1). This phenomenon requires time (usually $12 \mathrm{~h}$ ) but can be accelerated by the addition of parasite lysate or acetonitrile, lipidic extracts, corroborating the hypothesis of a determining role of lipids in $\beta$-hematin nucleation. The inhibition of $\beta$ hematin formation by a molecule is interpreted as a direct indication of its antimalarial activity. Inhibition can be quantified in several ways. Most of them require the separation of crystallized $\beta$-hematin from hematin monomers and amorphous aggregates, followed by monitoring of ${ }^{14} \mathrm{C}$-hematin incorporation into $\beta$-hematin or spectrophotometric measurement of free hematin absorbance after $\beta$-hematin dissolution, sometimes following pyridine addition.

The $\beta$-hematin test has been implemented to HTS format. In such approach, the hit yield depends on the nature of the library and cannot be used to evaluate the method efficiency to detect antimalarial compounds. Instead, the hit-to-lead ratio between the number of hits detected in the screening and the number of compounds actually validated as antimalarials by the subsequent in vitro parasite growth test is of significant value. A low hit-to-lead ratio reflects a significant amount of false positives. In two reported HTS campaigns, one using parasitederived reagents and radiolabeled hematin, ${ }^{26}$ another using the pyridine-spectrophotometric method, ${ }^{27}$ the hit-to-lead ratio was around 9\% (100 000 compounds, active hit defined by an $\mathrm{IC}_{50}$ $>5 \mu \mathrm{M}$ ) and 3\% (16000 compounds, active hit defined by an $\left.\mathrm{IC}_{50}>20 \mu \mathrm{M}\right)$, respectively.

Besides their poor hit-to-lead ratio, a salient feature of tests based on $\beta$-hematin formation is that heme must have the ferric state (Fe(III) heme). Indeed, no $\beta$-hematin formation occurs with the $\mathrm{Fe}$ (II) heme or in the presence of reducing agents. ${ }^{4,28,29}$ Therefore, the mechanism of action of artemisinine, the most active current antimalarial, does not take place in such conditions, and assays based on $\beta$-hematin formation cannot detect artemisinin-like compounds. As a matter of fact, the antimalarial activity of artemisinin has been discovered empirically through the traditional use of Artemisia annua by Chinese herbalists. Its modern use as a pure compound had to wait a thousand years before being pointed out by Chinese researchers focusing on the antimalarial activity of the plant. Other screening tests have been developed, on the basis of other heme detoxication pathways, but do not have the ability to detect artemisinin. ${ }^{30}$

Finally, the $\beta$-hematin assay does not provide any information on the structure of a compound showing inhibition. This means that, if an active compound contains impurities or if it is a complex mixture, it has to be either purified or processed by the so-called bioguided fractionation. For natural extracts, this implies the tedious isolation of the active compound and the risk of activity loss along with extract fractionation. However, the potential of biodiversity for malaria drug discovery is obvious. ${ }^{31-36}$ The huge extracts libraries available in companies or academic institutions require an access to HTS techniques but are renowned to be very difficult to handle in such approaches. ${ }^{37}$ Strategies combining HTS and techniques providing data simultaneously on activity and structures are still lacking.

The present method may be of significant interest for such research areas. Its application to an automatized, miniaturized pilot test was performed to assess its potential for HTS screening. Two 96-well plates totaling 172 compounds were tested out of our in-house library, consisting of pure compounds of natural or synthetic origin stored in a randomized order. Among these compounds, 18 were identified as forming adduct with heme. With the exception of one compound not tested for technical reasons, these compounds were tested on two Plasmodium strains, to assess growth inhibition. Among these compounds, 13 caused over 50\% inhibition at $10 \mu \mathrm{M}$ on the chloroquine-sensitive F32 strain and 8 caused over $50 \%$ inhibition at $10 \mu \mathrm{M}$ on the $\mathrm{K} 1$ chloroquineresistant strain. The hit-to-lead ratio can therefore be calculated as $76 \%$ and $47 \%$, respectively. Although these data have to be confirmed on a larger scale, the MS-based antiplasmodial screening shows promising to efficiently point out relevant compounds for further in vitro testing.

\section{CONCLUSIONS}

We developed a method based on mass spectrometry to detect and characterize compounds able to bind heme. As usual, screening tools based on $\beta$-hematin crystallization inhibition, the proposed screening method, is not devoted to directly correlate to an activity in vivo but is based on the interaction of small molecules with heme which is the main mechanism of action of most efficient known atimalarials. The method is consistent with literature data gathered for $\beta$-hematin inhibition. Validation on a serial of antimalarial drugs suggests that it can assess the binding strength between heme and molecule. It can specifically indicate the type of binding involved, allowing one to especially to detect artemisinin-like compounds. The artemisinin mechanism based on covalent binding to heme has proven to be highly effective to cure malaria, but rising resistance to artemisinin may require one to orient drug discovery to find structures differing significantly from artemisinin but based on the same mechanism of action. The present method fulfills this need and may be therefore of great interest in malaria drug discovery. The method is particularly interesting for screening purposes, as it has proved to be transferable to a HTS scale. Furthermore, it is worth noting that current assays based on $\beta$-hematin polymerization inhibition do not provide any information on the structure of a hit. The present method can detect a heme-binding compound within a complex mixture and simultaneously give structural information on the compound, opening the path for biological dereplication of natural extracts.

\section{ASSOCIATED CONTENT}

\section{S Supporting Information}

Data table from adducts and their stability curves, figures from chemical structures of cited compounds and from adducts obtained from extracts, and material and methods. This material is available free of charge via the Internet at http:// pubs.acs.org.

\section{AUTHOR INFORMATION}

\section{Corresponding Author}

*E-mail: bruno.figadere@u-psud.fr (B.F.); alexandre.maciuk@ u-psud.fr (A.M.).

\section{Present Addresses}

${ }^{\perp}$ Research coordinator, Research Center on Nutrition, Health and Wellness, Vidarium, Grupo Empresarial Nutresa, Medellin, ${ }^{\text {II }}$ Colombia. 
IIU.P. de Química Inorgánica y Orgánica, Universitat Jaume I, E-12080 Castellón, Spain.

"Research and Development Laboratory, Herb Pharm, 20260 Williams Hwy., Williams, Oregon 97544.

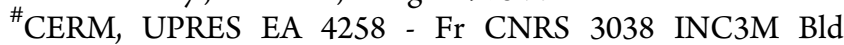
Becquerel, 14032 Caen cedex.

\section{Notes}

The authors declare no competing financial interest.

\section{ACKNOWLEDGMENTS}

K.M.-D. was supported by COLCIENCIAS, Colombia. K.M.D. and K.S. acknowledge the European Community's Sixth Framework Program for a Marie Curie Early Stage Training Fellowship, contract BioMedChem. We thank Prof Thomas Morton (University of California at Riverside, USA) and Karine Leblanc for valuable discussions and Audrey Solgadi from the Service d'Analyse des Médicaments et Métabolites (SAMM), the analytical platform of IPSIT 141, for her input and her assistance in performing triple quadrupole spectra acquisitions.

\section{REFERENCES}

(1) Wells, T. N. C. Science 2010, 329 (5996), 1153.

(2) Weissbuch, I.; Leiserowitz, L. Chem. Rev. 2008, 108, 4899-4914.

(3) Klonis, N.; Dilanian, R.; Hanssen, E.; Darmanin, C.; Streltsov, V.; Deed, S.; Quiney, H.; Tilley, L. Biochemistry-US 2010, 49, 6804-6811.

(4) Tekwani, B. L.; Walker, L. A. Comb. Chem. High Throughput Screening 2005, 8, 63-79.

(5) Corrêa Soares, J. B. R.; Menezes, D.; Vannier-Santos, M. A.; Ferreira-Pereira, A.; Almeida, G. T.; Venancio, T. M.; VerjovskiAlmeida, S.; Zishiri, V. K.; Kuter, D.; Hunter, R.; Egan, T. J.; Oliveira, M. F. PLoS Neglected Trop. Dis. 2009, 3 (7), No. e477.

(6) Wright, A. D.; Wang, H. Q.; Gurrath, M.; Konig, G. M.; Kocak, G.; Neumann, G.; Loria, P.; Foley, M.; Tilley, L. J. Med. Chem. 2001, 44, 873-885.

(7) Jonckers, T. H. M.; van Miert, S.; Cimanga, K.; Bailly, C.; Colson, P.; De Pauw-Gillet, M. C.; van den Heuvel, H.; Claeys, M.; Lemiere, F.; Esmans, E. L.; Rozenski, J.; Quirijnen, L.; Maes, L.; Dommisse, R; Lemiere, G. L. F.; Vlietinck, A.; Pieters, L. J. Med. Chem. 2002, 45, 3497-3508.

(8) Bilia, A. R.; Lazari, D.; Messori, L.; Taglioli, V.; Temperini, C.; Vincieri, F. F. Life Sci. 2002, 70, 769-778.

(9) Dascombe, M. J.; Drew, M. G. B.; Morris, H.; Wilairat, P.; Auparakkitanon, S.; Moule, W. A.; Alizadeh-Shekalgourabi, S.; Evans, P. G.; Lloyd, M.; Dyas, A. M.; Carr, P.; Ismail, F. M. D. J. Med. Chem. 2005, 48, 5423-5436.

(10) Pashynska, V. A.; Van den Heuvel, H.; Claeys, M. J. Am. Soc. Mass Spectrom. 2004, 15, 1181-1190.

(11) Kumar, S.; Guha, M.; Choubey, V.; Maity, P.; Bandyopadhyay, U. Life Sci. 2007, 80, 813-828.

(12) Chong, C. R.; Sullivan, D. J. Biochem. Pharmacol. 2003, 66, 2201-2212.

(13) Huy, N.; Kamei, K.; Kondo, Y.; Serada, S.; Kanaori, K.; Takano, R.; Tajima, K.; Hara, S. J. Biochem. 2002, 131, 437-444.

(14) Paunescu, E.; Susplugas, S.; Boll, E.; Varga, R.; Mouray, E.; Grosu, I.; Grellier, P.; Melnyk, P. ChemMedChem 2009, 4, 549-561.

(15) Hawley, S. R.; Bray, P. G.; Mungthin, M.; Atkinson, J. D.; O'Neill, P. M.; Ward, S. A. Antimicrob. Agents Chemother. 1998, 42, 682-686.

(16) Gemma, S.; Campiani, G.; Butini, S.; Joshi, B. P.; Kukreja, G.; Coccone, S. S.; Bernetti, M.; Persico, M.; Nacci, V.; Fiorini, I.; Novellino, E.; Taramelli, D.; Basilico, N.; Parapini, S.; Yardley, V.; Croft, S.; Keller-Maerki, S.; Rottmann, M.; Brun, R.; Coletta, M.; Marini, S.; Guiso, G.; Caccia, S.; Fattorusso, C. J. Med. Chem. 2009, 52, $502-513$.

(17) Held, J.; Westerman, R.; Kremsner, P. G.; Mordmuller, B. Antimicrob. Agents Chemother. 2010, 54, 540-542.
(18) Sweeney, D.; Raymer, M. L.; Lockwood, T. D. Biochem. Pharmacol. 2003, 66, 663-677.

(19) Pradines, B.; Tall, A.; Ramiandrasoa, F. R.; Spiegel, A.; Sokhna, C.; Fusai, T.; Mosnier, J.; Daries, W.; Trape, J. F.; Kunesch, G.; Parzy, D.; Rogier, C. J. Antimicrob. Chemother. 2006, 57, 1093-1099.

(20) Nzila, A. Drug Discovery Today 2006, 11, 939-944.

(21) Tarun, A. S.; Vaughan, A. M.; Kappe, S. H. I. Trends Parasitol. 2009, 25, 545-550.

(22) Fennell, B. J.; Naughton, J. A.; Dempsey, E.; Bell, A. Mol. Biochem. Parasitol. 2006, 145, 226-238.

(23) Xu, X. M.; Moller, S. G. Antioxid. Redox Signaling 2011, 15, 271-307.

(24) Robert, A.; Coppel, Y.; Meunier, B. Inorg. Chim. Acta 2002, 339, 488-496.

(25) Accardo, A.; Laurent, S. A. L.; Mazarguil, H.; Meyer, M.; Robert, A.; Meunier, B. J. Inorg. Biochem. 2007, 101, 1739-1747.

(26) Kurosawa, Y.; Dorn, A.; Kitsuji-Shirane, M.; Shimada, H.; Satoh, T.; Matile, H.; Hofheinz, W.; Masciadri, R.; Kansy, M.; Ridley, R. G. Antimicrob. Agents Chemother. 2000, 44, 2638-2644.

(27) Rush, M. A.; Baniecki, M. L.; Mazitschek, R.; Cortese, J. F.; Wiegand, R.; Clardy, J.; Wirth, D. F. Antimicrob. Agents Chemother. 2009, 53, 2564-2568.

(28) Tripathi, A. K.; Garg, S. K.; Tekwani, B. L. Biochem. Biophys. Res. Commun. 2002, 290, 595-601.

(29) Kannan, R.; Kumar, K.; Sahal, D.; Kukreti, S.; Chauhan, V. S. Biochem. J. 2005, 385, 409-418.

(30) Garavito, G.; Monje, M.-C.; Maurel, S.; Valentin, A.; Nepveu, F.; Deharo, E. Exp. Parasitol. 2007, 116, 311-313.

(31) de Villiers, K. A.; Egan, T. J. Molecules 2009, 14, 2868-2887.

(32) Fattorusso, E.; Taglialatela-Scafati, O. Mar. Drugs 2009, 7, 130152.

(33) Batista, R.; Júnior, A. d. J. S.; de Oliveira, A. B. Molecules 2009, 14, 3037-3072.

(34) Oliveira, A. B.; Dolabela, M. F.; Braga, F. C.; Jacome, R. L. R. P.; Varotti, F. P.; Povoa, M. M. An. Acad. Bras. Cienc. 2009, 81, 715-740.

(35) Kaur, K.; Jain, M.; Kaur, T.; Jain, R. Bioorg. Med. Chem. 2009, $17,3229-3256$.

(36) Calderon, L. D.; Silva-Jardim, I.; Zuliani, J. P.; Silva, A. D. E.; Ciancaglini, P.; da Silva, L. H. P.; Stabeli, R. G. J. Brazil. Chem. Soc. 2009, 20, 1011-1023.

(37) Koehn, F. E. In Natural compounds as drugs, Vol. i; Petersen, F., Amstutz, R., Eds.; Birkhaüser Verlag AG: Basel, 2008; Vol. 65, pp $175-210$. 